\title{
Recurrent nephrogenic adenoma in bladder diverticulum: case report and literature review
}

\author{
Rafael Boscolo-Berto, MD, FEBU; ${ }^{*+}$ Claudio Lamon, MD; ${ }^{+}$Mario Gardi, MD; ${ }^{; \pm}$Roberto Vezzaro, MD;* \\ Marina Gardiman, $M D^{\S}$
}

\begin{abstract}
*Department of Environmental Medicine and Public Health, Section of Legal Medicine and Forensic Pathology, University of Padova, Italy; 'Department of Oncological and Surgical Sciences, Urology

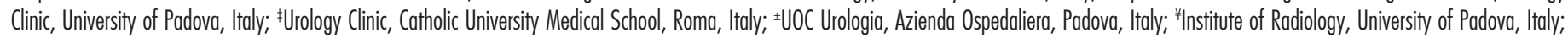
¿Department of Pathology, University of Padova, Italy
\end{abstract}

Cite as: Can Urol Assoc J 2012;6(2):e34-37. http://dx.doi.org/10.5489/cuaj.10140

\section{Abstract}

Nephrogenic adenoma is an uncommon benign lesion of the urinary tract induced by chronic irritation of the vesical mucosa, due to infection, trauma, surgery, calculi, foreign bodies and chemical agents. A 68-year-old male was admitted to our linic for a periodical cystoscopic evaluation as part of a follow-up initiated due to a past transitional cell carcinoma. The scheduled cystoscopy revealed, within a bladder diverticulum, an unexpected and completely asymptomatic nephrogenic adenoma that we removed by transurethral resection. We followed up the patient at 24 months, then later we made the diagnosis of nephrogenic adenoma. During this time, the patient experienced three relapses within the same diverticulum, always involving a nephrogenic adenoma we persistently treated by transurethral resections. As the nephrogenic adenoma is considered a benign lesion without any direct evidence of a possible evolution to an overt cancer, we successful attempted a half-yearly cystoscopic follow-up to control the growth of a highly recurrent benign entity, interposing between controls a periodical imaging. This paper represents the second report of a nephrogenic adenoma within a bladder diverticulum, but the first case of a nephrogenic adenoma highly recurrent within the same diverticulum and managed conservatively by regular transurethral resection scheduled over the time.

\section{Introduction}

Nephrogenic adenoma is an uncommon lesion of the urinary tract induced by chronic irritation of the vesical mucosa, due to infection, trauma, surgery, calculi, foreign bodies and chemical agents. ${ }^{1}$ Nephrogenic adenoma (also named nephrogenic metaplasia) is a benign entity and a common mimic of adenocarcinoma; this presents a diagnostic challenge in the interpretation of bladder biopsies. ${ }^{2}$

Davis issued the first report in 1949. ${ }^{3}$ The term "nephrogenic adenoma" was introduced a few years later by Friedman and Kuhlenbeck because the structure of neph- rogenic adenoma resembled that of a renal tubule. ${ }^{4}$

This paper represents the second report of a nephrogenic adenoma within a bladder diverticulum, but the first case of a nephrogenic adenoma highly recurrent within the same diverticulum and managed conservatively by regular scheduled transurethral resections.

\section{Case report}

A 68-year-old male was admitted to our clinic to undergo a periodical cystoscopic evaluation as an endoscopic followup for a transitional cell carcinoma (TCC) of the bladder. The patient's TCC was classified as non-muscle invasive at a transurethral resection of bladder (TUR-B) and nonrelapsing about 10 years earlier (1973 WHO [World Health Organization] classification T1G2). At the time of the first diagnosis of transitional bladder cancer, the patient refused the adjuvant intravesical instillation with a chemotherapic agent and chose a strictly endoscopic surveillance, which resulted in repeated negative investigations for about a decade.

During his follow-up, the patient underwent a transurethral resection of prostate (TURP) which confirmed benign prostatic hyperplasia. The patient also experienced multiple episodes of low urinary tract infections due to recurrent urethral strictures involving the bulbar portion and treated by endoscopic urethrotomy.

At the periodical follow-up imaging preceding our yearly endoscopic evaluation, the abdominopelvic computed tomography $(\mathrm{CT})$ underlined the presence of a bladder diverticulum stuffed by newly formed tissue (Fig. 1, panel A), without any grade of gross hematuria. A cystoscopic evaluation on the suspicion of a recurrence of TCC revealed a narrow necked, left bladder diverticulum that appeared to be partially filled with a greyish papillary tumour of about $1.5 \mathrm{~cm}$ with a large base of implant, similar to the picture of lowgrade TCC (Fig. 1, panel B). During the procedure, the polypoid lesion was entirely resected and a contextual extensive 

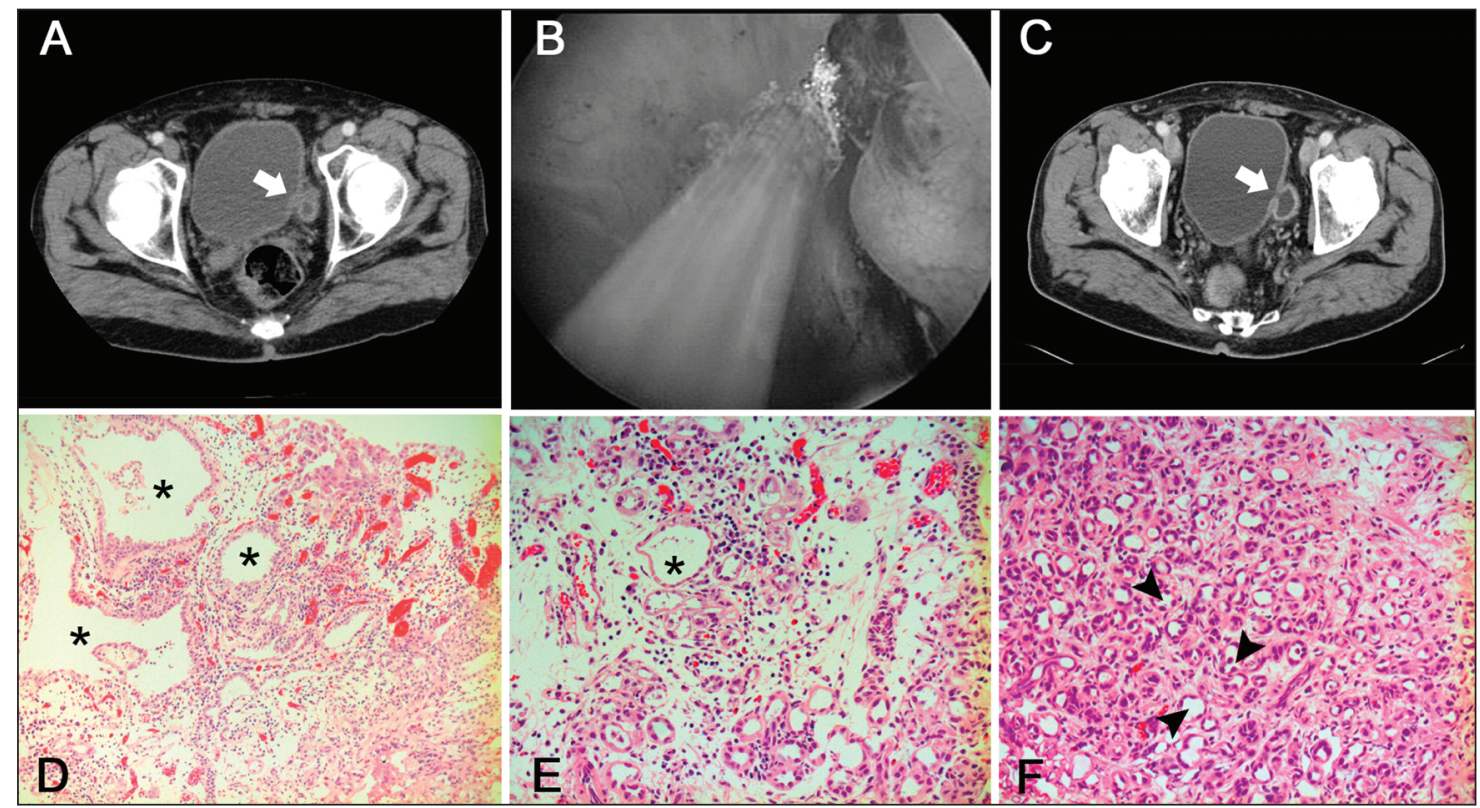

Fig. 1. A: abdominopelvic computed tomography (CT) with contrast medium evidencing the presence of a left bladder diverticulum stuffed by newly formed tissue (arrow). B: cystoscopic evaluation revealing the presence of a narrow necked, left bladder diverticulum partially filled with a greyish papillary tumor of about $1.5 \mathrm{~cm}$ with a large base of implant. C: last abdominopelvic CT with contrast medium, demonstrating no masses involving the bladder wall or spreading into the perivisceral tissue, and a regular profile of the bladder with the exception of the known actually free-of-disease diverticulum (arrow). D: tubular-glandular structures with a pseudocystic appearance $\left(^{*}\right)$ and lined with a single layer of cuboidal/cylindrical epithelium (Hematoxylin \& Eosin [H\&E], 100x). E: dilated tubule resembling a pseudocyst $\left(^{*}\right)$ containing conglomerates of mucin (H\&E, 200x). F: tubules lined by hobnail cells (arrows) showing focal atypias due to a degenerative process (H\&E, 200x).

coagulation on its base of implantation was performed. After the completion of this manoeuvre, a careful visual inspection confirmed the removal of the whole tumour, without macroscopic residuals or perforation involving the bladder diverticulum. Therefore, a transurethral catheter was placed for a day, and after its removal the patient was discharged.

The histopathological evaluation assessed the presence of a nephrogenic adenoma. This was characterized by a pseudoinfiltrative appearance of tubular-glandular structures with a pseudocystic appearance, lined by a single layer of cuboidal epithelium with a hobnail appearance and focal atypias due to a degenerative process (Fig. 1, panels D, E and F). Due to this benign finding, and the patient's refusal to undergo a surgical procedure, we opted for a periodical evaluation by cystoscopy combined with radiological investigation (mostly ultrasound), without any additional intervention.

We followed up the patient 24 months after the initial diagnosis of nephrogenic adenoma. During this time, the patient relapsed in the same diverticulum as nephrogenic adenoma at 2, 6 and 10 months, always presenting with only small residual abnormal tissue without any clinical disturbance. On imaging, there was no evidence of masses involving the bladder wall or spreading into the perivisceral tissue, confirming the regular bladder profile, with the exception of the known and actually free-of-disease diverticulum, as demonstrated by the last abdominopelvic CT (Fig. 1, panel C).

\section{Discussion}

The nephrogenic adenoma constitutes an infrequent benign metaplasia of the urothelial mucosa, probably underestimated in literature. ${ }^{5}$ It could be recognized in most of cases within the bladder, but reports indicated it could also rarely be detected in other sites, such as the renal pelvis, ureter and bladder exstrophy, urethral diverticula, in an ileal conduit and in the bowel portion of an augmented bladder. ${ }^{1}$

In our case, bladder diverticula are essentially pseudodiverticula formed by mucosal herniations through areas of acquired weakness in the muscular bladder wall as a result of increased intravesical pressure secondary to bladder outlet obstruction. Carcinomas arising in diverticula account for about $0.8 \%$ to $10 \%$ of all carcinomas arising in the bladder. ${ }^{6,7}$ Only a limited number of papers have examined the histopathological features of tumours arising within bladder diverticula, reporting the presence of TCC, squamous cell carcinoma, adenocarcinoma and sarcomatoid carcinoma. ${ }^{6}$

Our clinical case represents the fourth report of a nephrogenic adenoma within a bladder diverticulum, ${ }^{8,9}$ but the first one on a nephrogenic adenoma highly recurrent within the same diverticulum and managed conservatively by regular transurethral resection of the bladder (TUR-B).

The etiology of nephrogenic adenoma is not clear, however, most authors agree that patients have been exposed 
Boscolo-Berto et al.

to some kind of chronic irritation or inflammation due to some predisposing factors, such as trauma, chronic infection, previous surgery, urinary stones, irradiation and urinary catheterization or repeated instrumentation for diagnostic/ therapeutic purposes. ${ }^{1}$ The latter is our case, as the patient underwent a TURP and multiple episodes of endoscopic urethrotomy.

The endoscopic appearance of nephrogenic adenoma could be polypoid, papillary or flat. They were mostly localized on the bladder wall; this is generally limited to lesions less than $1 \mathrm{~cm}$ and up to $7 \mathrm{~cm}$, and are rarely reported as a burden disease. ${ }^{1,10}$ The macroscopic appearance of the lesion varies, but is generally highly vascularized, which justifies the appearance of the disease with hematuria microor macroscopic. ${ }^{10}$ However, our patient represents a rare case of incidental diagnosis of such a condition, as he was totally asymptomatic.

The initial visual diagnosis, using cystoscopy, could raise suspicion of transitional cancer, as nephrogenic adenoma appears similar to low-grade TCC. Therefore, the definite diagnosis of nephrogenic adenoma is based on pathologic analysis, resulting from biopsy and histological examination of the lesion. ${ }^{11}$

A TUR-B of the lesions and subsequent extensive coagulation of their base is generally considered the treatment of choice for accurate diagnosis and symptom relief of all recurrent lesions. ${ }^{1,12}$

In the literature, a high recurrence rate (37.5\% to $75 \%)$ of nephrogenic adenoma has been found during long-term follow-up of pediatric patients. ${ }^{10,13}$ Our patient confirms this; he experienced three recurrent episodes over 24 months. Nevertheless, despite such a frequent recurrence, there are no reports indicating that nephrogenic adenoma transforms to a malignant or metastatic lesion over the time. ${ }^{1,4,10}$ The other previous report on nephrogenic adenoma within a bladder diverticulum was treated by bilateral pelvic lymphadenectomy and bladder diverticulectomy. ${ }^{8}$ At that time, there was limited knowledge on the behaviour of this condition over time and the diverticulum was filled by the adenoma. ${ }^{8}$ Subsequent reports gradually advanced the chance of a conservative treatment by periodical TUR-B to successfully manage limited lesions. Some follow-up arrangements were proposed to monitor these kinds of patients, by different timing of the cystoscopic evaluations. ${ }^{1,10,14}$

Diverticular resection of a bladder cancer represents an additional challenge in assessing the real depth of tumour invasion, because of the anatomical structure of the bladder wall at that location lacks a conventional muscularis propria. In this setting, an extensive deep sampling of the diverticular wall is prohibitive and increases the risk of bladder perforation.

Moreover, the paucity of muscle fibres beyond the mucosa theoretically allows malignant tumours to invade the perivesical fat earlier and more easily than in a normal bladder wall, making it difficult to stratify the tumours into superficial and invasive bladder cancer. ${ }^{15,16}$ Nevertheless, as the invasion beyond the lamina propria into the muscular layer results exclusively from aggressive tumour biology and not from a mere anatomical contiguity, it is comprehensible why low-grade, biologically indolent tumours and benign histological entities can be managed conservatively despite the lack of a muscular layer beyond the mucosa, provided the tumours have been completely removed. ${ }^{7}$

\section{Conclusion}

The atypical nephrogenic metaplasia is recognized as benign with the occasional substantial cytological abnormalities of no apparent clinical significance. There is also no direct evidence that links atypical nephrogenic metaplasia to cancer. ${ }^{2}$ Due to the benign nature of this lesion, patients do not adhere to the frequent cystoscopic controls. ${ }^{10}$ As such, we believe that a half-yearly cystoscopic evaluation is sufficient to control the growth of a highly recurrent benign entity, interposing between controls the careful ultrasound evaluation of a well-filled bladder and periodical urinary cytology.

Competing interests: None declared.

This paper has been peer-reviewed.

\section{References}

1. Porcaro $A B, D^{\prime} A m i c o A$, Ficarra $V$, et al. Nephrogenic adenoma of the urinary bladder: our experience and review of the literature. Urol Int 2001;66:152-5. http://dx.doi.org/10.1159/000056596

2. Cheng L, Cheville IC, Sebo TJ, et al. Atypical nephrogenic metaplasia of the urinary tract: a precursor lesion? Cancer 2000;88:853-61. htrp://dx.doi.org/10.1002/(SICI) 1097-0142(20000215)88:4<853::AIDCNCR16>3.0.C0;2-H

3. Davis TA. Hamartoma of the urinary bladder. Northwest Med 1949;48:182-5.

4. Friedman NB, Kuhlenbeck H. Adenomatoid tumors of the bladder reproducing renal structures (nephrogenic adenomas). J Urol 1950;64:657-70.

5. Boscolo-Berto, R. Clinical testing and evidence-based medicine: when the absence of evidence doesn't mean evidence of absence [in Italian]. G Ital Nefrol 2009;26:417.

6. Tamas EF, Stephenson AJ, Campbell SC, et al. Histopathologic features and clinical outcomes in 71 cases of bladder diverticula. Arch Pathol Lab Med 2009;133:791-6.

7. Golijanin D, Yossepowitch O, Beck SD, et al. Carcinoma in a bladder diverticulum: presentation and treatment outcome. J Urol 2003;170:1761-4. hitp://dx.doi.org/10.1097/01.ju.0000091800.15071.52

8. Heffernan JP, Huisman TK. Nephrogenic adenoma in a bladder diverticulum. J Urol 1994;152:1208-10.

9. Tanase K, Tawada M, Muranaka K, et al. Two cases of nephrogenic adenoma in the bladder diverticulum [in Japanese]. Hinyokika Kiyo 2000;46:815-7.

10. Chen CS, Cheng CL. Nephrogenic adenoma of the urinary bladder: clinical experience and review of the literature. J Chin Med Assoc 2006;69:166-8. http://dx.doi.org/10.1016/S1726-4901 (09)70199-6

11. Zougkas K, Kalafatis M, Kalafatis P. Nephrogenic adenoma of the urinary bladder. Int Urol Nephrol 2004;36:513-7. http://dx.doi.org/10.1007/s11255-004-0848-7

12. Peeker R., Aldenborg F, Fall M. Nephrogenic adenoma-a study with special reference to clinical presentation. Br J Urol 1997;80:539-42. http://dx.doi.org/10.1046/i.1464-410X.1997.00418.x

13. Husain AN, Armin AR, Schuster GA. Nephrogenic metaplasia of urinary tract in children: report of three cases and review of the literature. Pediatr Pathol 1988;8:293-300. http://dx.doi. org/10.3109/15513818809042972 
Recurrent vesical nephrogenic adenoma

14. Navarre RJ Jr, Loening SA, Platz C, et al. Nephrogenic adenoma: a report of 9 cases and review of the literature. J Urol 1982; 127:775-9.

15. Redman JF, McGinnis TB, Bissada NK. Management of neoplasms in vesical diverticula. Urology 1976;7:492-4. http://dx.doi.org/10.1016/0090-4295(76)90186-2

16. Rubin P. Current concepts in genitourinary oncology: a multidisciplinary approach. J Urol 1971;106:31538.
Correspondence: Dr. Rafael Boscolo-Berto, Department of Environmental Medicine and Public Health, Section of Legal Medicine and Forensic Pathology, Department of Oncological and Surgical Sciences, Urology Clinic, University of Padova, Via Falloppio 50, 35128 Padova, Italy; fax: 0039 0497969541; rafael.boscoloberto@unipd.it 